\title{
Anti-SARS-CoV-2 Nucleoprotein Antibodies Derived from Pig Serum with a Controlled Specificity
}

\author{
Jaeyong Jung ${ }^{1}$ Ji-Hong Bong ${ }^{1} \cdot$ Hong-Rae Kim ${ }^{1} \cdot$ Jun-Hee Park ${ }^{1}$ Chang Kyu Lee ${ }^{1}$ Min-Jung Kang ${ }^{2} \cdot$ Hyun Ok Kim $^{3}$. \\ Jae-Chul Pyun ${ }^{1}$ (1)
}

Received: 1 February 2021 / Revised: 1 February 2021 / Accepted: 11 March 2021 / Published online: 25 March 2021

(c) The Korean BioChip Society 2021

\begin{abstract}
Antibodies against severe acute respiratory syndrome coronavirus 2 (SARS-CoV-2) nucleoprotein (NP) were purified from pig serum through two steps: (1) isolation of anti-NP IgG antibodies using magnetic beads with immobilized human SARSCoV-2 NP and (2) filtration of anti-spike protein (SP) IgG antibodies using magnetic beads with immobilized human SARS-CoV SP. The enhanced specificity of the purified antibodies to the NP of SARS-CoV-2 was demonstrated using an immunoassay with anti-NP IgG antibodies after the isolation and filtration steps. The binding constants $\left(K_{\mathrm{d}}\right)$ of the purified anti-NP IgG antibodies to the NP of SARS-CoV-2 and the SP of SARS-CoV were estimated using a surface plasmon resonance biosensor (SPR). A competitive assay using the two-step purified anti-NP IgG antibodies from pig serum demonstrated (a) the detection of SARS-CoV-2 in viral fluid and (b) the discrimination of SARS-CoV-2 from SARS-CoV, MERS-CoV, and $\mathrm{CoV}$ strain 229E in viral fluids.
\end{abstract}

Keywords SARS-CoV-2 $\cdot$ SARS-CoV $\cdot$ Nucleoprotein $(\mathrm{NP}) \cdot$ Spike protein $(\mathrm{SP}) \cdot$ Immunoassay

\section{Introduction}

Recently, we reported that the antibodies against the nucleoprotein (NP) of severe acute respiratory syndrome coronavirus 2 (SARS-CoV-2) could be isolated from pig serum and the antibodies could be used for the detection of SARS$\mathrm{CoV}-2$ in viral culture fluid [1]. Coronavirus ( $\mathrm{CoV})$ has been classified into four genera, including alpha-, beta-, gamma-, and delta-CoV. The CoV strains causing severe acute respiratory syndromes such as SARS-CoV-2 of COVID-19, SARS-CoV, and MERS-CoV, belong to beta-CoV genera $[2,3]$. Alpha- and beta-CoV genera are known to infect

Jae-Chul Pyun

jcpyun@yonsei.ac.kr

1 Department of Materials Science and Engineering, Yonsei University, 50 Yonsei-Ro, Seodaemun-Gu, Seoul 03722, Republic of Korea

2 Korea Institute of Science and Technology (KIST), 5 Hwarang-ro 14-gil, Seongbuk-gu, Seoul 02792, Republic of Korea

3 Department of Laboratory Medicine, Yonsei University College of Medicine, 50-1 Yonsei-Ro, Seodaemun-Gu, Seoul 03722, Republic of Korea humans and other animals $[4,5]$. In pigs, several types of $\mathrm{CoV}$ infections occur, such as those due to pig epidemic diarrhea virus (PEDV; alpha-CoV), transmissible gastroenteritis virus (TGEV; alpha-CoV), and pig hemagglutinating encephalomyelitis virus (PHEV; beta-CoV) $[6,7]$. From homology analysis of the amino acid sequences, the NP of SARS-CoV-2 and the above-mentioned CoVs of pigs show a high similarity (identity) of $40 \%$ (27\%) to PEDV (Uniprot ID: Q07499), 48\% (28\%) to TGEV (Uniprot ID: P04134), and 55\% (40\%) to PHEV (Uniprot ID: Q8BB23). Thus, pig serum is considered to contain antibodies against these $\mathrm{CoVs}$ from infection history, and these antibodies in pig serum are considered to have a cross-affinity to the NP of SARS-CoV-2 [1]. Thus, in this study, isolated anti-NP $\mathrm{IgG}$ antibodies were a polyclonal mixture and these antibodies could be used for the detection of SARS-CoV-2 as well as other $\mathrm{CoV}$ strains such as SARS-CoV, MERS-CoV, and CoV strain 229E in viral culture fluids using a competitive immunoassay configuration [8] .

From the competitive assay, the isolated anti-NP IgG antibodies were estimated to have a similar affinity to SARS-CoV-2 and SARS-CoV, and these antibodies showed a relatively low affinity to MERS-CoV and CoV strain $229 \mathrm{E}$ in viral culture fluids. For selective detection 
of SARS-CoV-2, affinity enhancement was required. In this study, the isolation of antibodies against SARS-CoV-2 was accomplished by removing the antibodies with affinity to SARS-CoV from the polyclonal antibody mixture [9]. Antibodies were isolated from pig serum through two steps: (Step 1) isolation of anti-NP IgG antibodies using magnetic beads with immobilized nucleoprotein (NP) of SARS-CoV-2 and (Step 2) filtration to remove antibodies with SARS-CoV affinity using magnetic beads with immobilized SARS-CoV spike protein (SP) (Fig. 1a) [10]. The enhanced specificity of the isolated antibodies to the NP of SARS-CoV-2 was observed using an immunoassay with anti-NP IgG antibodies after Step 1 and after Step $1+$ Step 2 . The binding constants $\left(\mathrm{K}_{\mathrm{d}}\right)$ of anti-NP $\mathrm{IgG}$ antibodies to the NP of SARS-CoV-2 and SP of SARS-CoV after Step 1 and after Step $1+$ Step 2 were estimated using a surface plasmon resonance biosensor. A competitive assay using anti-NP IgG antibodies obtained from pig serum through two-step purification demonstrated (a) detection of SARS-CoV-2 in viral fluid and (b) discrimination of SARS-CoV-2 from SARS-CoV, MERS-CoV, and CoV strain 229E in viral fluids.

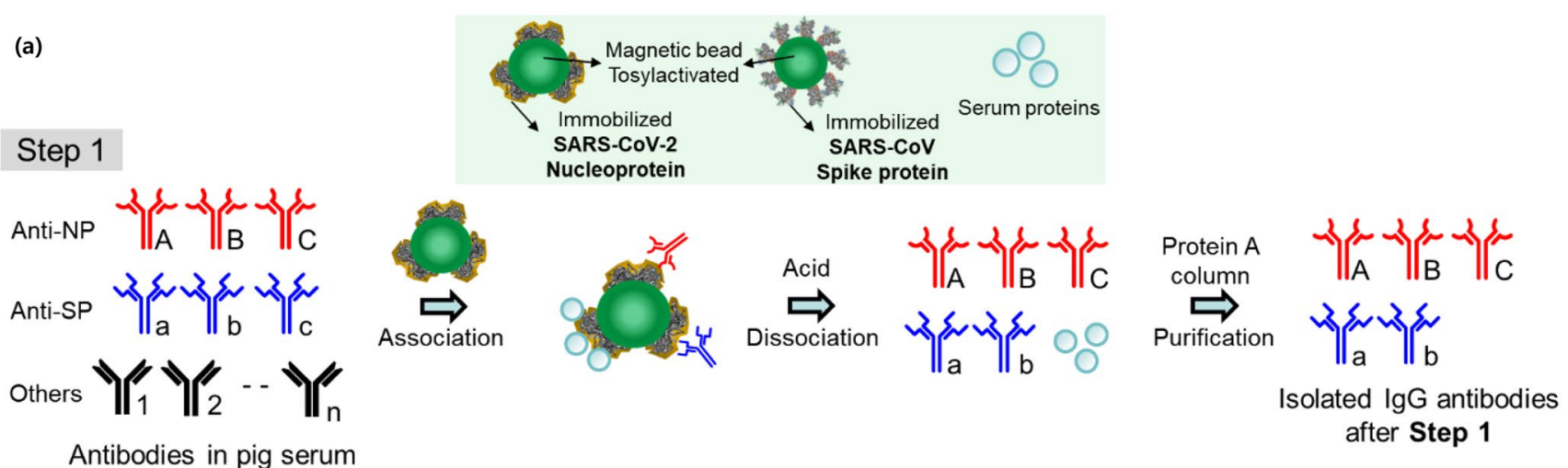

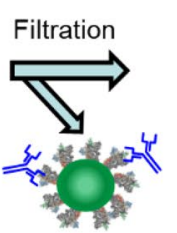

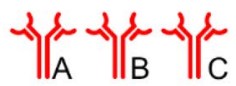

Filtered anti-NP IgG antibodies

after Step $1+$ Step 2 (b)

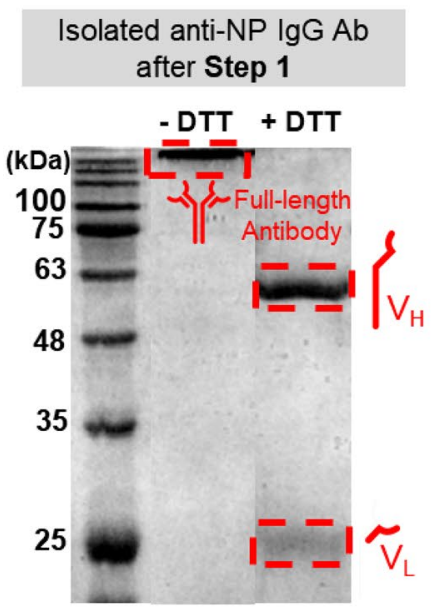

Filtered anti-NP IgG Ab

after Step 1 + Step 2

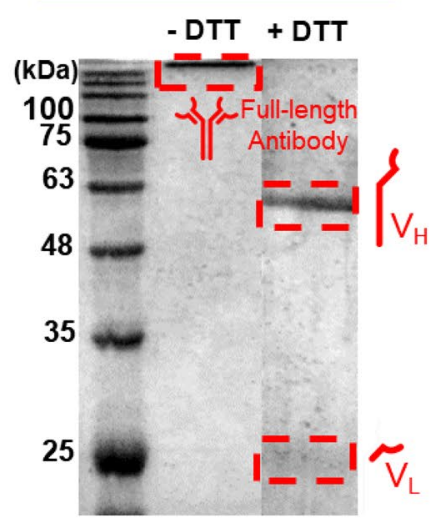

Fig. 1 Isolation of anti-nucleoprotein of SARS-CoV-2 (NP) IgG antibodies from pig serum. a The anti-NP IgG antibodies were first isolated using magnetic beads with immobilized NP (Step 1), and then the filtration was carried out using magnetic beads with immobilized
SP (Step 2). b SDS-PAGE of the isolated antibodies after Step 1 and after Step 1+Step 2 under non-reducing/reducing conditions without/ with dithiotreitol (DTT) 


\section{Results and Discussion}

\subsection{Purification of Anti-NP IgG Antibodies}

As previously mentioned, pig serum is known to contain antibodies against proteins from various $\mathrm{CoVs}$, because pigs have been infected by several kinds of alpha- and betaCoVs, such as PEDV (alpha-CoV), TGEV (alpha-CoV), and PHEV (beta-CoV) [6]. The amino acid sequences of the NPs of these CoVs known to infect pigs were analyzed and showed significant similarities of more than $40-55 \%$ compared with the nucleoprotein (NP) of human SARS-CoV-2 [1]. Thus, anti-NP IgG antibodies were isolated from pig serum, and the isolated antibodies were reported to have a specificity to NP of human SARS-CoV-2. However, the pigserum-derived anti-NP IgG antibodies also showed a similar specific binding affinity to the viral culture fluids of human SARS-CoV. CoVs are known to produce four types of major proteins, including envelope proteins, spike proteins, membrane proteins, and nucleoproteins. Among these proteins, nucleoprotein and spike proteins are known to be produced most abundantly, and these proteins have been frequently used for vaccine development and assays [11-15]. In this study, the isolation of anti-NP IgG antibodies specific to SARS-CoV-2 was enhanced using a two-step purification method. A precondition for this method is that the isolated anti-NP IgG antibodies must be part of a polyclonal mixture of antibodies. As shown in Fig. 1a, anti-NP IgG antibodies were first isolated using magnetic beads with immobilized NP, which contained anti-NP IgG antibodies as well as antiSP IgG antibodies. Among the isolated antibodies, the antibodies with affinity to SP were filtered out using magnetic beads with immobilized SP. Finally, anti-NP IgG antibodies with enhanced specificity to the SARS-CoV-2 NP could be obtained, because the anti-SP IgG antibodies were removed by the filtering step.

The isolated antibodies are shown in Fig. 1b. SDSPAGE showed that the isolated antibodies from Step 1 and Step $1+$ Step 2 were immunoglobulin G (IgG) comprising heavy chains $(50 \mathrm{kDa})$ and light chains $(25 \mathrm{kDa})$. Moreover, the area of antibody protein bands decreased by as much as $32 \%$ based on densitometric analysis [16, 17]. The yield of antibody isolation was estimated to be $8.37 \pm 0.53 \mu \mathrm{g}$ per $1 \mathrm{ml}$ of pig serum after Step 1 and $5.71 \pm 0.18 \mu \mathrm{g}$ per $1 \mathrm{ml}$ of pig serum after Step $1+$ Step 2 .

\subsection{Binding Properties of Purified Anti-NP IgG Antibodies}

The binding properties of anti-NP IgG antibodies after Step 1 and after Step $1+$ Step 2 were analyzed using an immunoassay. The binding properties of anti-NP IgG antibodies after Step 1 were analyzed separately using microplates with immobilized NP of SARS-CoV-2 and immobilized SP of SARS-CoV (Fig. 2a). The quantity of bound antibodies was analyzed using horseradish peroxidases (HRP)-labeled secondary antibodies and the chromogenic reaction of 3,3',5,5'-tetra-methylbenzidine (TMB) [18]. The relative binding intensity of antibodies after Step 1 was estimated to be $43.1 \%$ to SP of SARS-CoV compared with $100 \%$ to NP of SARS-CoV-2 (Fig. 2b). The same immunoassay was performed using anti-NP IgG antibodies after Step $1+$ Step 2 using magnetic beads with immobilized SP. The relative binding intensity of antibodies after Step $1+$ Step 2 was estimated to be $9.2 \%$ to SP of SARS-CoV compared with $100 \%$ to NP of SARS-CoV-2 (Fig. 2c). From the comparison of relative binding intensities, the anti-NP IgG antibodies collected after the filtration step showed that the binding affinity to the SP of SARS-CoV decreased from $43.1 \%$ (Step 1) to $9.2 \%$ (Step $1+$ Step 2) compared with the binding affinity to the NP of SARS-CoV-2 (100\%). These results showed that the isolation step (Step 1) followed by the filtration step (Step 2) could enhance the specificity of the anti-NP IgG antibodies by decreasing the relative amount of anti-SP IgG antibodies.

The binding properties of anti-NP IgG antibodies after Step 1 and after Step 1+ Step 2 were analyzed separately using an Au-SPR chip with immobilized NP of SARSCoV-2 and immobilized SP of SARS-CoV (Fig. 3a). The quantity of bound antibodies was analyzed at a range of $37-1000 \mathrm{ng} / \mathrm{mL}$ antibody concentration from the difference in baselines between initial and after dissociation of antibodies from the Au-SPR chip. The binding constant $\left(\mathrm{K}_{\mathrm{d}}\right)$ of antibodies after Step 1 was estimated to be $2.3 \times 10^{-9} \mathrm{M}$ to the SP of SARS-CoV and $1.5 \times 10^{-9} \mathrm{M}$ to the NP of SARS-CoV-2 (Fig. 3b). The same immunoassay was performed using anti-NP antibodies after the filtration step (Step 2). The binding constant $\left(\mathrm{K}_{\mathrm{d}}\right)$ of antibodies after Step $1+$ Step 2 was estimated to be $3.4 \times 10^{-9} \mathrm{M}$ to the SP of SARS-CoV and $9.9 \times 10^{-10} \mathrm{M}$ to the NP of SARSCoV-2 (Fig. 3c). Based on the binding constants, the antiNP IgG antibodies after the filtration step showed that the binding affinity to the SP of SARS-CoV decreased by as much as $43 \%$, whereas that to the NP of SARS-CoV-2 increased by as much as $63 \%$ compared with the antibodies after Step 1. These results also showed that the isolation step (Step 1) followed by the filtration step (Step 2) could enhance the specificity of the anti-NP IgG antibodies by decreasing the relative amount of anti-SP IgG antibodies. 
Fig. 2 Binding properties of the isolated anti-NP IgG antibodies after Step 1 and after Step $1+$ Step 2. a Configuration for the binding activity test using immobilized NP on microplate. Comparison of binding affinities of the isolated anti-NP IgG antibodies after $\mathbf{b}$ Step $1(n=3)$ and c Step $1+$ Step $2(n=3)$ against nucleoprotein (NP) of SARS$\mathrm{CoV}-2$ and spike protein (SP) of SARS-CoV (a)

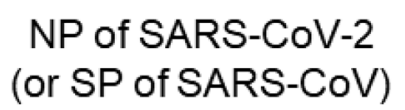

\section{BSA blocking}

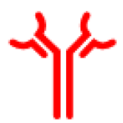

Anti-NP lgG antibody

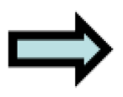

Maxiplate
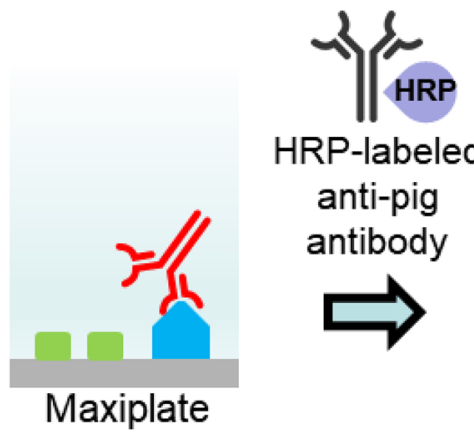

HRP-labeled anti-pig antibody
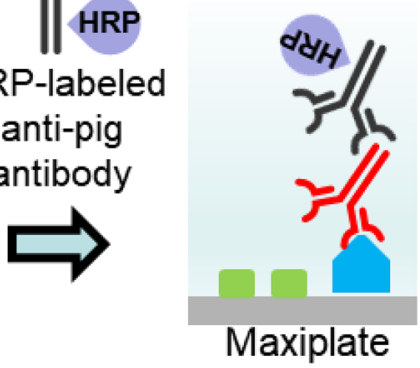

(b)

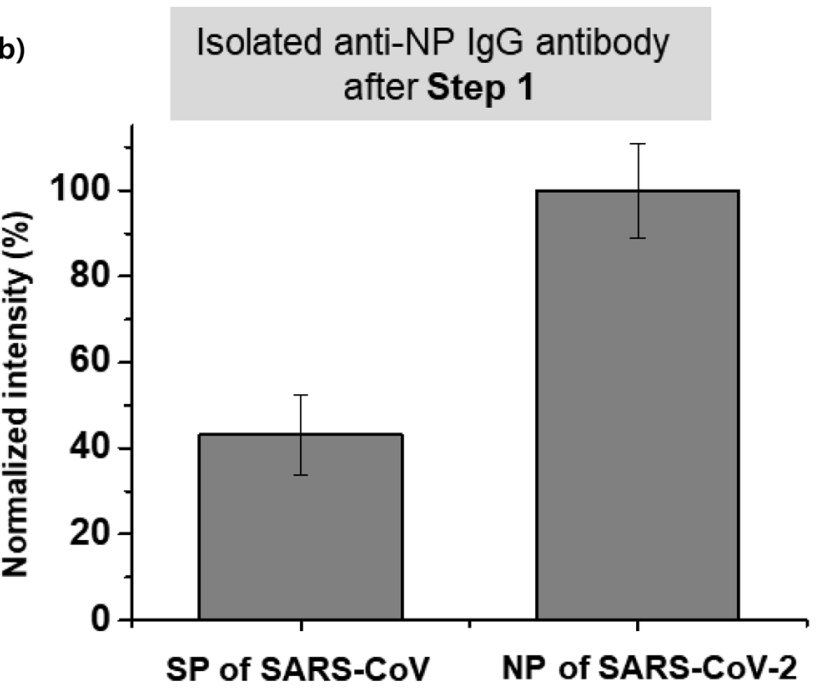

(c)

Filtered anti-NP IgG antibody after Step 1 + Step 2

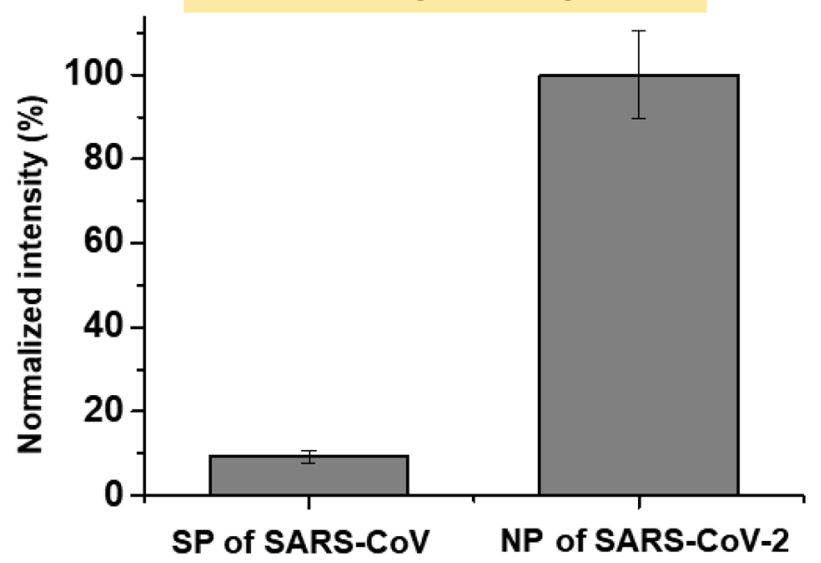

\subsection{Competitive Assay Using Purified Anti-NP Antibodies}

Anti-NP IgG antibodies from the two-step purification were applied in a competitive assay of various CoVs in viral culture fluids. The competitive assay was performed by mixing a sample with an assay solution comprising antiNP IgG antibodies and magnetic beads with immobilized 
(a)
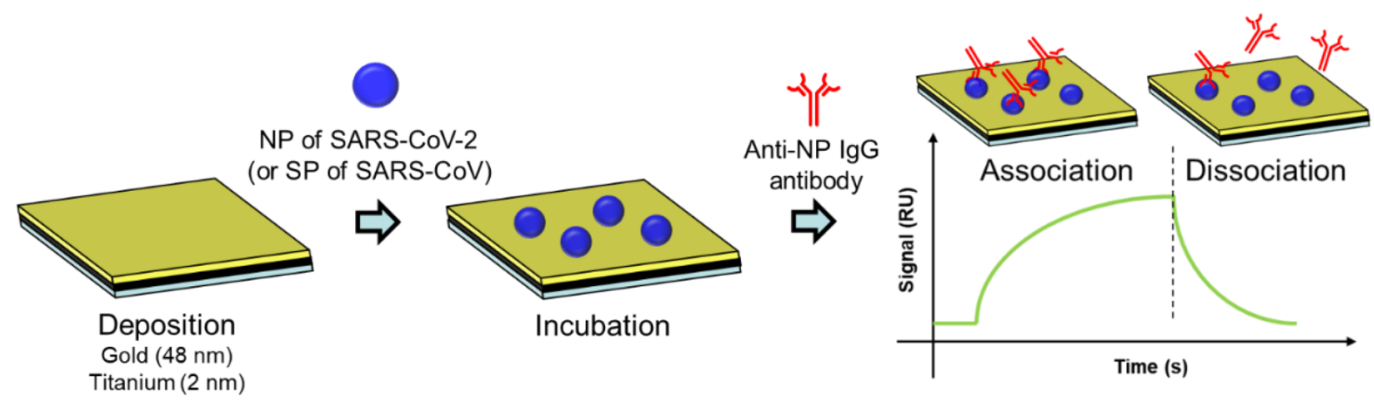

(b)
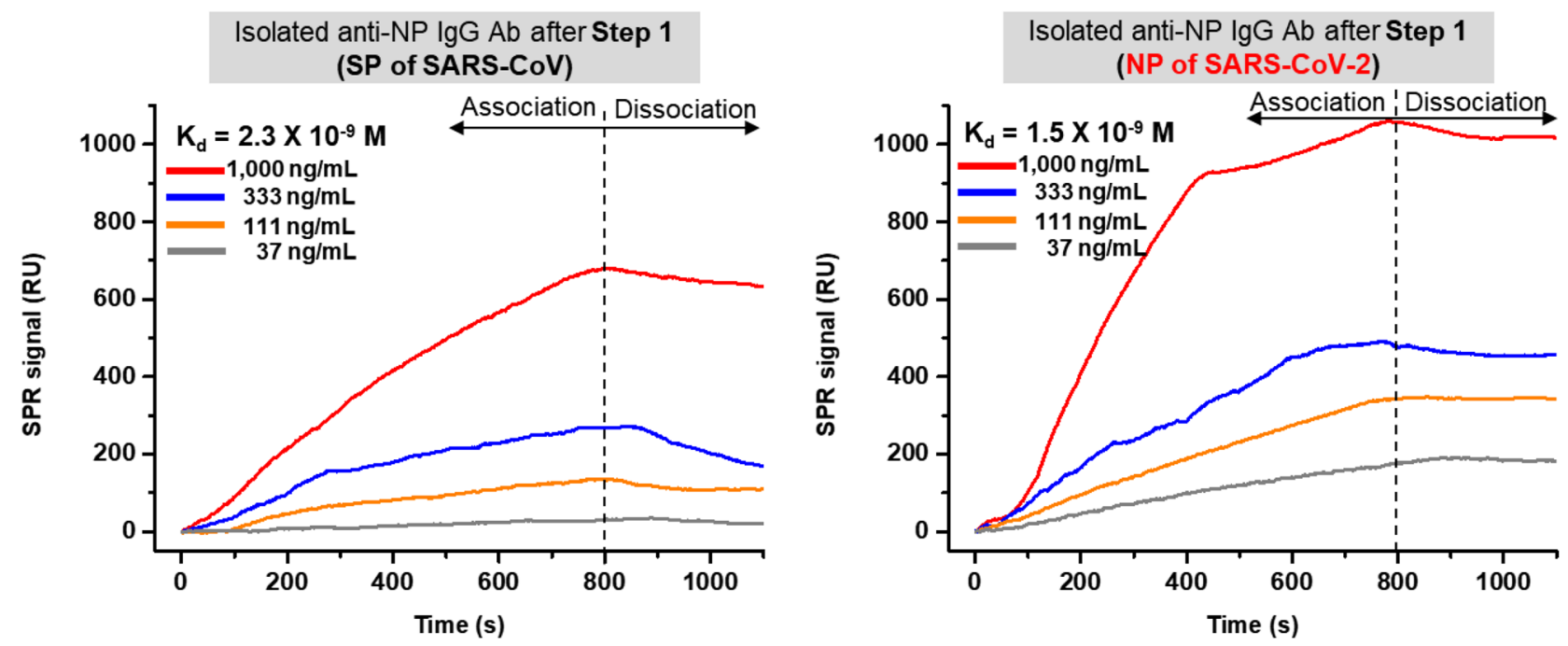

(c)

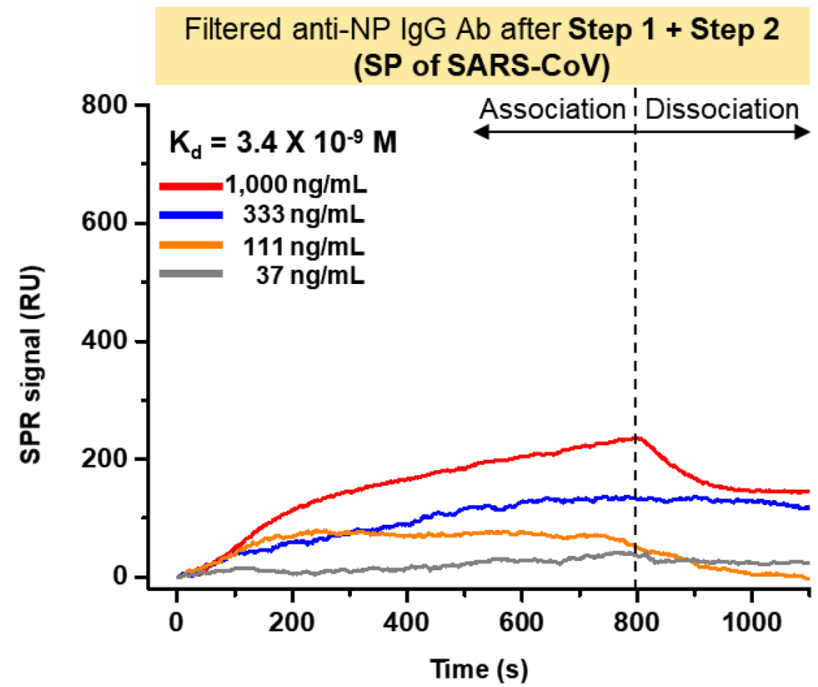

Fig. 3 Estimation of binding constants $\left(K_{\mathrm{d}}\right)$ of the isolated anti-NP IgG antibodies using SPR biosensor. a Configuration for the SPR measurement using immobilized SP of SARS-CoV and NP of SARS-

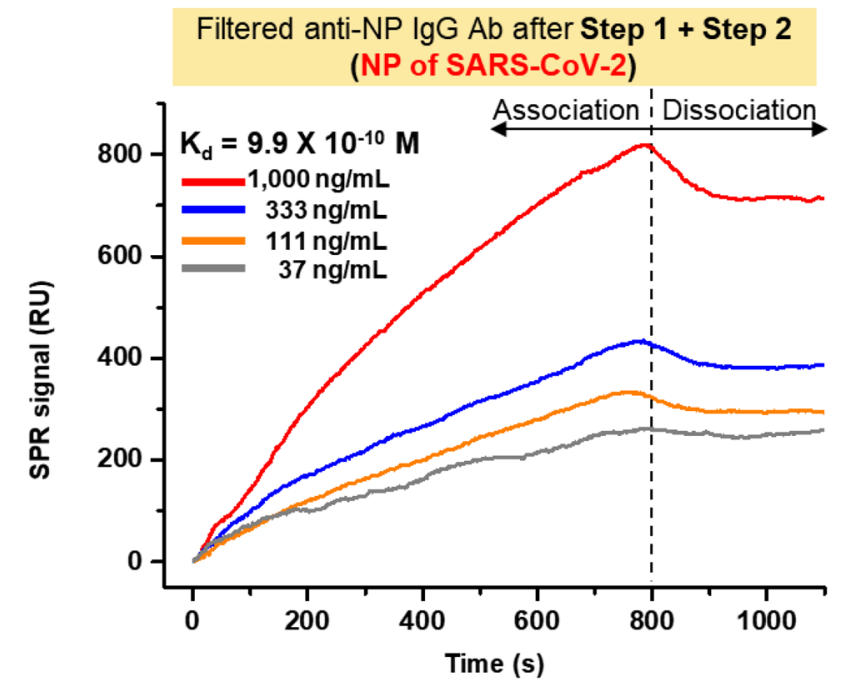

CoV-2 on Au-chip. Binding constants $\left(K_{\mathrm{d}}\right)$ of the isolated anti-NP IgG antibodies after b Step 1 and $\mathbf{c}$ Step $1+$ Step 2 against NP of SARS-CoV-2 and SP of SARS-CoV 
(a)

Positive sample

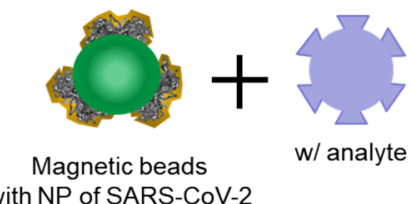

with NP of SARS-CoV-2

Negative control

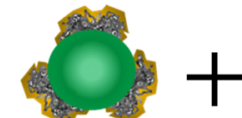

Magnetic beads with NP of SARS-CoV-2

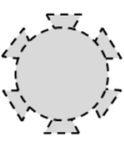

w/o analyte
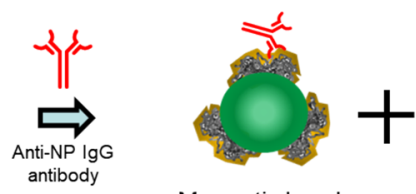

Magnetic beads with NP of SARS-CoV-2

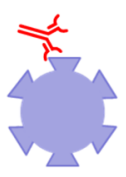

w/ analyte w/ analyte

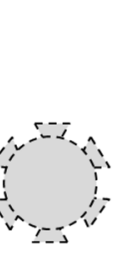

w/o analyte
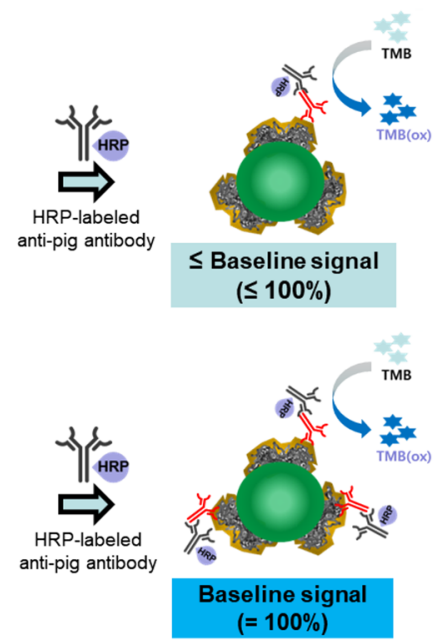

(b) Isolated anti-NP IgG antibody after Step 1

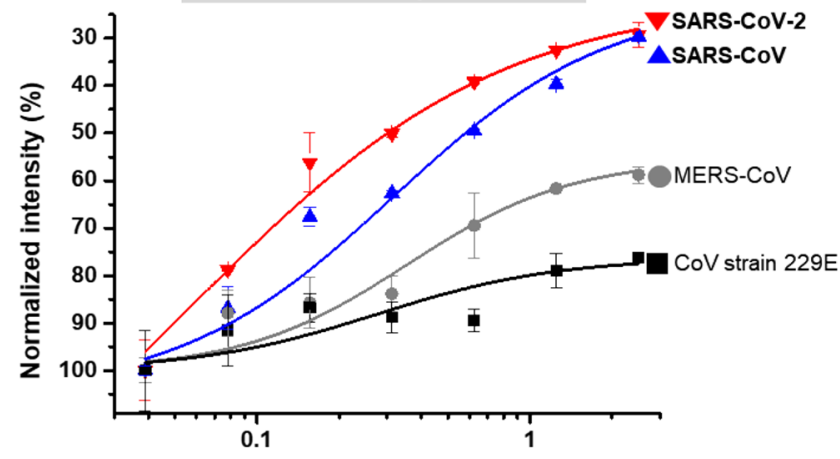

Fraction of culture fluid (\%)

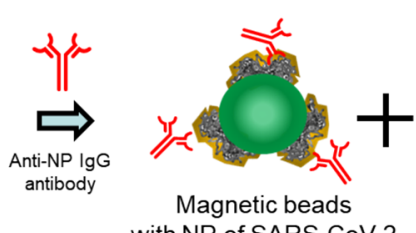

with NP of SARS-CoV-2
Fig. 4 Competitive assay of CoV viral culture fluids of SARS-CoV-2, SARS-CoV, MERS-CoV, CoV strain 229E using the isolated anti-NP $\mathrm{IgG}$ antibodies. a Configuration for the competitive assay using magnetic beads with immobilized NP and the isolated anti-NP IgG anti-

NP at known concentrations (Fig. 4a). In the negative control without NP of SARS-CoV-2, the mixed anti-NP $\mathrm{IgG}$ antibodies in the assay solution were bound only to the magnetic beads with immobilized NP. The quantity of bound antibodies on the magnetic beads was evaluated using secondary antibodies labeled with HRP and TMB as chromogenic substrates. In the case of positive samples with NP of SARS-CoV-2, the NP in the sample reacted with the anti-NP IgG antibodies and the remaining antibodies bound to the magnetic beads. Because of competition with the mixed anti-NP IgG antibodies, the quantity of bound anti-NP IgG antibodies on the magnetic beads was less than that of the negative control. From the chromogenic reaction of secondary antibodies labeled with $\mathrm{HRP}$ and TMB, the optical density at $450 \mathrm{~nm}\left(\mathrm{OD}_{450 \mathrm{~nm}}\right)$ decreased according to the amount of NP in the sample, as shown in Fig. 4b and c. The amount of NP in the sample

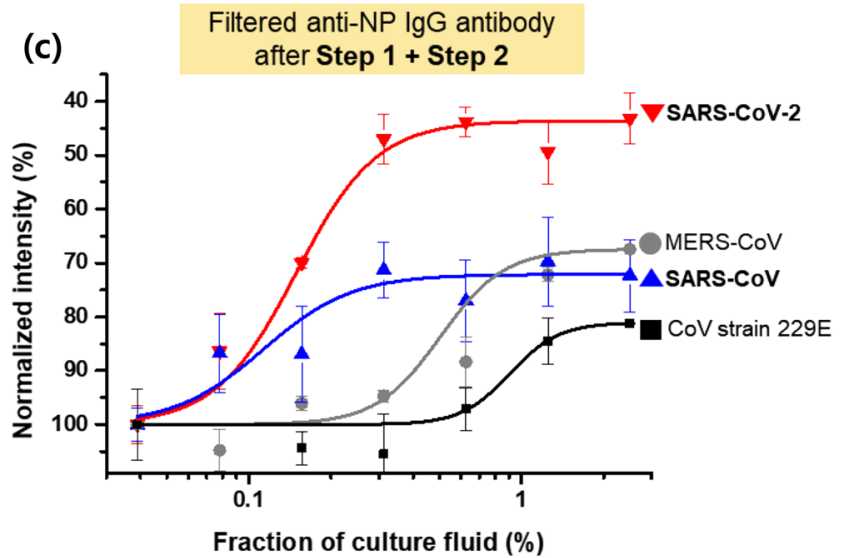

bodies at a known concentration. Results of the competitive assay for four kinds of $\mathrm{CoV}$ viral culture fluids using the isolated anti-NP IgG antibodies after b Step $1(n=3)$ and $\mathbf{c}$ Step $1+\operatorname{Step} 2(n=3)$

could be estimated based on the difference between the $\mathrm{OD}_{450 \mathrm{~nm}}$ of each sample and the negative control.

When the anti-NP IgG antibodies from the isolation step (Step 1) were used for the competitive assay, standard curves were obtained for four viral culture fluids including SARSCoV-2, SARS-CoV, MERS-CoV, and CoV strain 229E (Fig. 4b). The viral culture fluids were diluted from 40-fold (fraction of $2.5 \%$ ) to 1280 -fold (fraction of $0.08 \%$ ) with PBS. As the fraction of culture fluid increased or as the NP concentration increased, the normalized intensity decreased for all four viral culture fluids. In particular, the difference in signal was significantly greater for SARS-CoV-2 and SARS-CoV samples than for the other two culture fluids, demonstrating the high specificity of the antibodies to NP in SARS-CoV-2 and SARS-CoV samples. Additionally, the signals from the competitive assays for SARS-CoV-2 and SARS-CoV samples were quite similar, demonstrating the 
Table 1 Comparison of amino acid sequence of nucleoprotein (NP) of CoV strains

\begin{tabular}{lll}
\hline Compared viral species & Similarity (\%) & Identity (\%) \\
\hline $\begin{array}{l}\text { SARS-CoV-2 NP versus SARS-CoV } \\
\quad \text { NP }\end{array}$ & 94 & 91 \\
$\begin{array}{l}\text { SARS-CoV-2 NP versus MERS-CoV } \\
\quad \text { NP }\end{array}$ & 63 & 48 \\
SARS-CoV-2 NP versus CoV 229E NP & 40 & 28 \\
\hline
\end{tabular}

low specificity of the antibodies in discriminating between the NP of SARS-CoV-2 and SARS-CoV in samples. As summarized in Table 1, such similarities in the assay results were considered to be because of the homology of amino acid sequences between the NP of SARS-CoV-2 and the three other CoV strains.

Anti-NP IgG antibodies after the two-step purification were used for the competitive assay, and standard curves were obtained for four viral culture fluids including SARSCoV-2, SARS-CoV, MERS-CoV, and CoV strain 229E, as shown in Fig. 4c. When the viral culture fluids were diluted using the same range as the previous experiment, the difference in normalized intensity was also decreased for all four viral culture fluids. The difference in signal was significantly greater for SARS-CoV-2 samples than for the other three culture fluids using the two-step purified anti-NP antibodies, demonstrating a high specificity of the antibodies to the NP of SARS-CoV-2. Additionally, the signals from the competitive assays for SARS-CoV-2 and SARS-CoV samples were at a significantly level, demonstrating the high specificity of the antibodies in discriminating between the NPs of SARSCoV-2 and SARS-CoV.

\section{Conclusions}

Specific anti-NP IgG antibodies against SARS-CoV-2 were isolated from pig serum. The antibodies were purified using a two-step method: (Step 1) isolation of anti-NP IgG antibodies using magnetic beads with immobilized NP and (Step 2) filtration of anti-SP IgG antibodies from previously isolated antibodies using magnetic beads with immobilized SP. The yield of antibody isolation was estimated to be $8.37 \pm 0.53 \mu \mathrm{g}$ per $1 \mathrm{ml}$ of pig serum after Step 1 and $5.71 \pm 0.18 \mu \mathrm{g}$ per $1 \mathrm{ml}$ of pig serum after Step $1+$ Step 2 . Analysis of the binding properties of anti-NP IgG antibodies following the two-step purification showed enhanced specificity of the anti-NP IgG antibodies to SARS-CoV-2 by decreasing the relative amount of anti-SP IgG antibodies. The binding constant $\left(\mathrm{K}_{\mathrm{d}}\right)$ of antibodies after Step 1 was estimated to be $2.34 \times 10^{-9} \mathrm{M}$ to the SP of SARS-CoV and $1.54 \times 10^{-9} \mathrm{M}$ to the NP of SARS-CoV-2 using an SPR biosensor. Additionally, the binding constant $\left(K_{\mathrm{d}}\right)$ of antibodies after Step $1+$ Step 2 was estimated to be $3.36 \times 10^{-9} \mathrm{M}$ to the SP of SARS-CoV and $9.91 \times 10^{-10} \mathrm{M}$ to the NP of SARS$\mathrm{CoV}-2$. From the comparison of the binding constants, the anti-NP antibodies after the filtration step showed that the binding affinity to the SP of SARS-CoV was reduced by $43 \%$ and that to the NP of SARS-CoV-2 was increased by $63 \%$ relative to the antibodies from Step 1. Anti-NP IgG antibodies were applied in a competitive assay of various CoVs in culture fluids: SARS-CoV-2, SARS-CoV, MERS$\mathrm{CoV}$, and CoV strain 229E. For the competitive assay with the anti-NP IgG antibodies from the two-step purification process, the difference in signal was significantly greater for SARS-CoV-2 samples than for the other three culture fluids, demonstrating the high specificity of the antibodies to the NP in SARS-CoV-2 samples. Additionally, the signals from the competitive assays for SARS-CoV-2 and SARS$\mathrm{CoV}$ samples were at a significant level, demonstrating a high specificity of the antibodies in discriminating between the NPs of SARS-CoV-2 and SARS-CoV. The results show that isolated anti-NP IgG antibodies from this two-step purification process could be used for the detection of SARSCoV-2 and the discrimination of SARS-CoV-2 from other $\mathrm{CoV}$ strains in viral fluids.

\section{Materials and Methods}

\subsection{Materials}

HRP-labeled anti-pig IgG antibody (ab102135) was purchased from Abcam (Cambridge, UK). Tosyl-activated magnetic beads (diameter, $2.8 \mu \mathrm{m}$ ) were purchased from Invitrogen Co. (Carlsbad, CA, USA). Bicinchoninic acid (BCA) assay kit, Maxisorp microplates, pig serum, and TMB reagent were purchased from Thermo Fisher Scientific (Waltham, MA, USA). Other chemicals were purchased from Sigma-Aldrich Korea (Seoul, Korea). Culture fluids of SARS-CoV-2, SARS-CoV, MERS-CoV, and CoV strain of 229E were purchased from Zeptometrix (Buffalo, NY, USA). NP of SARS-CoV-2 and SP of SARS-CoV were supplied by Optolane (Seongnam, Korea) and Genbody (Cheonan, Korea).

\subsection{Purification of Anti-NP Antibodies}

Magnetic beads with immobilized NP of SARS-CoV-2 and SP of SARS-CoV were prepared by mild mixing of magnetic beads $(165 \mu \mathrm{L})$ at $5 \mathrm{mg} / \mathrm{mL}$ and NP or SP solution $(100$ $\mu \mathrm{L})$ at $1 \mathrm{mg} / \mathrm{mL}$ for $18 \mathrm{~h}$ at $37{ }^{\circ} \mathrm{C}$. Anti-NP IgG antibodies were isolated from pig serum as follows (Fig. 1). Step (1) Pig serum (1 mL) was reacted to magnetic beads with immobilized NP of SARS-CoV-2 by mild shaking with a wheel-rotating mixer (RT-10, Daehan Scientific Co, Wonju, 
Korea) at $10 \mathrm{rpm}$ for $1 \mathrm{~h}$. Proteins bound to the magnetic beads were dissociated by treatment with $0.1 \mathrm{M}$ glycine- $\mathrm{HCl}$ buffer ( $\mathrm{pH} 2.7,1 \mathrm{~mL}$ ) for $30 \mathrm{~s}$, and $1 \mathrm{M}$ Tris- $\mathrm{HCl}$ buffer $(\mathrm{pH}$ $10.5,20 \mu \mathrm{L}$ ) was added for neutralization. The antibody fraction from the neutralized solution was then separated using a protein-A column. Step (2) Purified anti-NP IgG antibodies were filtered using magnetic beads with immobilized SP of SARS-CoV. The prepared anti-NP IgG antibodies were concentrated with an Amicon filter with a cutoff pore size of $10 \mathrm{kDa}$. The yield of anti-NP IgG antibodies per $1 \mathrm{~mL}$ of pig serum was determined using a BCA assay kit according to the manufacturer's instructions.

\subsection{Binding Assay of Anti-NP Antibodies}

The binding properties of the isolated anti-NP IgG antibodies after Step 1 and after Step $1+$ Step 2 were estimated using a microplate binding assay, as shown in Fig. 2. The NP of SARS-CoV-2 and the SP of SARS-CoV solutions $(100 \mu \mathrm{L})$ at a concentration of $20 \mu \mathrm{g} / \mathrm{mL}$ were incubated in a Maxisorp microplate overnight at $4{ }^{\circ} \mathrm{C}$. After washing with $0.1 \%$ PBST and PBS, each well was blocked with bovine serum albumin at $5 \mathrm{mg} / \mathrm{mL}$ for $1 \mathrm{~h}$. After washing, $100 \mu \mathrm{L}$ of $1 \mu \mathrm{g} / \mathrm{mL}$ anti-NP IgG antibody solution was reacted for $1 \mathrm{~h}$ at room temperature. After washing with $0.1 \%$ PBST and PBS, $100 \mu \mathrm{L}$ of $20 \mathrm{ng} / \mathrm{mL}$ HRP-labeled secondary antibody solution was reacted for $1 \mathrm{~h}$. After washing with $0.1 \%$ PBST and PBS, the chromogenic reaction was performed using TMB reagent for $3 \mathrm{~min}$. After quenching with $2 \mathrm{M}$ sulfuric acid $(100 \mu \mathrm{L}), \mathrm{OD}_{450 \mathrm{~nm}}$ was measured using a microplate reader (Versamax, Molecular Devices, Sunnyvale, CA, USA).

\section{SPR Measurement}

The binding constants $\left(K_{\mathrm{d}}\right)$ of anti-NP IgG antibodies after Step 1 and after Step 1+ Step 2 were estimated using an SPR biosensor from i-ClueBio (Seongnam, Korea), as shown in Fig. 3. Au chips for SPR measurement were prepared by sputtering (1) an adhesive layer of titanium (2-nm thickness) and (2) a gold layer (48-nm thickness) on BK-7 glass $\left(10 \times 10 \mathrm{~mm}^{2} ; 0.5-\mathrm{mm}\right.$ thickness $)$ NP of SARS-CoV-2 and SP of SARS-CoV were immobilized on the SPR chip by incubation of each protein solution at $20 \mu \mathrm{g} / \mathrm{mL}$ for $1 \mathrm{~h}$. After washing with $0.1 \%$ PBST and PBS, anti-NP IgG antibody solution at a concentration range of $37-1000 \mathrm{ng} / \mathrm{mL}$ was continuously injected into the flow cell for $15 \mathrm{~min}$, and PBS was then injected continuously into the flow cell for $10 \mathrm{~min}$. The pumping rate was set to $15 \mu \mathrm{L} / \mathrm{min}$ for the association and dissociation steps. The binding constants $\left(K_{\mathrm{d}}\right)$ of anti-NP IgG antibodies after Step 1 and after Step $1+$ Step
2 were calculated using a fitting software (iMstart) supplied by i-ClueBio.

\subsection{Competitive Assay of Anti-SARS-CoV-2 Antibodies}

Furthermore, $10 \mu \mathrm{L}$ of $1 \mathrm{mg} / \mathrm{mL}$ magnetic beads with immobilized NP of SARS-CoV-2 and $10 \mu \mathrm{L}$ of $5 \mu \mathrm{g} / \mathrm{mL}$ anti-NP IgG antibodies were mixed as a reaction solution. Then, 20 $\mu \mathrm{L}$ reaction solution was mixed with various concentrations of each sample $(180 \mu \mathrm{L})$ by mild shaking for $1 \mathrm{~h}$. After isolation of magnetic beads with an external magnet, the magnetic beads were washed with $0.1 \%$ PBST and PBS. Subsequently, $150 \mu \mathrm{L}$ of $20 \mathrm{ng} / \mathrm{mL}$ HRP-labeled anti-pig IgG antibody solution was incubated with the magnetic beads for $1 \mathrm{~h}$, and the chromogenic reaction was performed using TMB reagent for $3 \mathrm{~min}$. After quenching with $2 \mathrm{M}$ sulfuric acid $(100 \mu \mathrm{L}), \mathrm{OD}_{450 \mathrm{~nm}}$ was measured using a microplate reader.

\subsection{Standard Curve Fitting}

The results of SPR signals were fitted using the four-parameter logistic equation [19, 20] as described below:

$y=\frac{a-d}{1+(x / c)^{b}}+d$

where $a$ and $d$ are the maximum and minimum signals (RU), respectively; $c$ is the concentration of anti-NP IgG antibody (nM); and $b$ is the Hill's slope of the curve.

Acknowledgements This work was supported by the National Research Foundation of Korea [Grant Numbers: NRF-2020R1A2B5B01002187 and NRF-2020R1A5A101913111].

\section{References}

1. Bong, J.-H., et al.: Pig sera-derived anti-SARS-CoV-2 antibodies in surface plasmon resonance biosensors. BioChip. J. 14, 358-368 (2020). https://doi.org/10.1007/s13206-020-4404-z

2. Monchatre-Leroy, E., et al.: Identification of alpha and beta coronavirus in wildlife species in France: bats, rodents, rabbits, and hedgehogs. Viruses. 9, 364 (2017). https://doi.org/10.3390/v9120 364

3. Wu, D., Wu, T., Liu, Q., Yang, Z.: The SARS-CoV-2 outbreak: what we know. Int. j. Infect. Dis. (2020). https://doi.org/10.1016/j. ijid.2020.03.004

4. Chen, Y., Liu, Q., Guo, D.: Emerging coronaviruses: genome structure, replication, and pathogenesis. J. Med. Virol. 92, 418 423 (2020). https://doi.org/10.1002/jmv.25681

5. Lu, X., et al.: SARS-CoV-2 infection in children. New Engl. J. Med. 382, 1663-1665 (2020). https://doi.org/10.1056/NEJMc 2005073

6. Kim, J.-M., et al.: Identification of Coronavirus Isolated from a Patient in Korea with COVID-19. Osong Public Health Res. Perspect. 11, 3 (2020). https://doi.org/10.24171/j.phrp.2020.11.1.02 
7. Elfiky, A.A.: Anti-HCV, nucleotide inhibitors, repurposing against COVID-19. Life Sci. 248, 117477 (2020). https://doi.org/10. 1016/j.lfs.2020.117477

8. Bong, J.-H., et al.: Competitive Immunoassay of SARS-CoV-2 Using Pig Sera-Derived Anti-SARS-CoV-2 Antibodies. BioChip. J. 15, 1-9 (2021). https://doi.org/10.1007/s13206-021-00011-6

9. Park, J.-H., et al.: Microbial biosensor for Salmonella using antibacterial antibodies isolated from human serum. Enzym. Microb. Technol. 144, 109721 (2021). https://doi.org/10.1016/j.enzmictec. 2020.109721

10. Ali, A., Vijayan, R.: Dynamics of the ACE2-SARS-CoV-2/SARS$\mathrm{CoV}$ spike protein interface reveal unique mechanisms. Sci. Rep. 10, 1-12 (2020). https://doi.org/10.1038/s41598-020-71188-3

11. Du, L., et al.: The spike protein of SARS-CoV-a target for vaccine and therapeutic development. Nat. Rev. Microbiol. 7, 226236 (2009). https://doi.org/10.1038/nrmicro2090

12. Amanat, F., Krammer, F.: SARS-CoV-2 vaccines: status report. Immunity (2020). https://doi.org/10.1016/j.immuni.2020.03.007

13. Ghodake, G.S., et al.: Biological characteristics and biomarkers of novel SARS-CoV-2 facilitated rapid development and implementation of diagnostic tools and surveillance measures. Biosens. Bioelectron. 177, 112969 (2021). https://doi.org/10.1016/j.bios. 2021.112969

14. Funari, R., Chu, K.-Y., Shen, A.Q.: Detection of antibodies against SARS-CoV-2 spike protein by gold nanospikes in an opto-microfluidic chip. Biosens. Bioelectron. 169, 112578 (2020). https:// doi.org/10.1016/j.bios.2020.112578

15. Kim, H.-Y., et al.: Development of a SARS-CoV-2-specific biosensor for antigen detection using $\mathrm{scFv}-\mathrm{Fc}$ fusion proteins. Biosens.
Bioelectron. 175, 112868 (2020). https://doi.org/10.1016/j.bios. 2020.112868

16. Bong, J.-H., et al.: Fluorescence immunoassay of E. coli using anti-lipopolysaccharide antibodies isolated from human serum. Biosens. Bioelectron. 126, 518-528 (2019). https://doi.org/10. 1016/j.bios.2018.10.036

17. Lee, G.-Y., et al.: Thermophoretic diagnosis of autoimmune diseases based on Escherichia coli with autodisplayed autoantigens. Anal. Chim. Acta. 1055, 106-114 (2019). https://doi.org/10. 1016/j.aca.2018.12.038

18. Kim, H.-R., et al.: An on-chip chemiluminescent immunoassay for bacterial detection using in situ-synthesized cadmium sulfide nanowires with passivation layers. BioChip. J. 14, 268-278 (2020). https://doi.org/10.1007/s13206-020-4305-1

19. Hasani, A., et al.: Recent progress of two-dimensional materials and metal-organic framework-based taste sensors. J. Korean Ceram. Soc. 57, 353-367 (2020). https://doi.org/10.1007/ s43207-020-00047-8

20. Kim, H.-R., et al.: Characterization of in-situ synthesized CdS x Se $1-x$ ternary alloy nanowire photosensor. J. Korean Ceram. Soc. 56, 308-316 (2019). https://doi.org/10.4191/kcers.2019.56.3.10

Publisher's Note Springer Nature remains neutral with regard to jurisdictional claims in published maps and institutional affiliations. 